\section{Cat-scratch disease in Crete: an update}

\section{Georgios Minadakis,}

Emmanouil Angelakis,

Dimosthenis Chochlakis, Yannis Tselentis,

Anna Psaroulaki

Laboratory of Clinical Bacteriology, Parasitology, Zoonoses and Geographical Medicine, WHO Collaborating Center for Mediterranean Zoonoses, University of Crete, Staurakia-Voutes, Crete, Greece

\section{Abstract}

There are few epidemiological and clinical studies about the presence of cat scratch disease (CSD) on the island of Crete. The objective of this study was to analyze a large number of patients with suspected CSD to define the frequency of Bartonella infections in Crete. From January 2005 to October 2008, we studied patients with suspected CSD from hospitals in Crete. Sera of the referred patients were tested by immunofluorescence assay (IFA). For some patients, we also received lymph nodes and blood samples that we tested for the presence of Bartonella henselae by molecular assays. Overall, we tested 507 serum samples and we found 56 (11\%) cases of CSD. PCR assay was positive for 2 patients; one had a $B$. henselae positive lymph node and the other a positive whole blood sample. Significantly more CSD cases $(62.5 \%$, 35 of 56) were reported in children than in infants and adults $(\mathrm{P}<0.05)$. Moreover, we identified that most cases of CSD occurred between May and September $(\mathrm{P}=0.002)$ and December and January. CSD is prevalent in Crete and is mostly associated with an increase in outdoor activity.

\section{Introduction}

Bartonella henselae is the main causative agent of cat scratch disease (CSD), which appears to be the most common organism responsible for lymphadenopathy in adults and children. ${ }^{1}$ Recently, $B$. henselae was also associated with a syndrome characterized by scalp eschar and neck lymphadenopathy following tick bites (SENLAT). ${ }^{2}$ Rarely, CSD has also been associated with $B$. quintana and recently with $B$. alsatica. ${ }^{3}$ Cats are the main reservoirs of $B$. henselae, and the bacterium is transmitted among cats by the cat flea (Ctenocephalides felis). ${ }^{1}$ However, epidemiological data do not support efficient transmis- sion from cat to human via the $C t$. felis ${ }^{4}$ and most case series of CSD patients report that symptoms developed after the patient received a scratch. ${ }^{5}$ The transmission of $B$. henselae from cat to human via a scratch is nevertheless a rare event, considering the number of pet cats and the frequency of cat scratches and bites inflicted by them.

Patients with classic CSD usually present a gradual regional lymph node enlargement, usually accompanied by a round, red-brown, non-tender papule, which develops in the line of the scratch after 3-10 days and may persist for only a few days or as long as 2-3 weeks. ${ }^{6}$ Recently, live $B$. henselae was found in the primary inoculation sites of 3 patients after a cat scratch. ${ }^{7}$ The enlarged lymph node is often painful and tender. ${ }^{6}$ Lymphadenopathy occurs in the nodes that drain the area where cat scratches usually occur; in the axilla, the neck, and the groin. ${ }^{8}$ The infection is usually selflimited, with the frequent development of extensive regional lymph node enlargement that typically lasts 2-3 months and occasionally longer. ${ }^{6}$ Most patients with typical CSD remain afebrile and are not systemically ill throughout the course of the disease. As a reference center for bartonelloses, we routinely receive samples from patients with suspected CSD. In this study, we analyzed a large number of patients with suspected CSD in Crete during a 3-year period. Our objective was to define the frequency of Bartonella infections and to characterize clinical and laboratory features.

\section{Materials and Methods}

\section{Patients}

From January 2005 to October 2008, we studied patients with suspected CSD from hospitals of the four prefectures of Crete: Chania (30 patients), Rethymno, ${ }^{14}$ Heraklion (446), and Lasithi. ${ }^{17}$ Clinico-epidemiological patient inclusion criteria were: fever over $38^{\circ} \mathrm{C}$ and/or lymphadenopathy and/or visual disturbance and/or contact with cat or fleas. A standardized questionnaire to collect this information, together with details of treatment, outcome, possible complications, etc., was completed for each patient. For each patient, we received at least one serum sample. For 122 patients we also received a convalescent-phase serum sample. For 10 patients, we also received a lymph node biopsy specimen and a whole blood sample. Patients were classified as definitely having CSD if there was direct evidence of infection with Bartonella sp. using culture, serology or molecular assays.

Serology

Sera of the referred patients were tested by
Correspondence: Anna Psaroulaki, Laboratory of Clinical Bacteriology, Parasitology, Zoonoses and Geographical Medicine. Voutes, Heraklion, Crete, 71100, Greece.

Tel. +30.281 .039 .4743 - Fax: +30.281 .039 .4740$

E-mail: annapsa@med.uoc.gr

Key words: Bartonella henselae, cat-scratch disease, Crete.

Conflict of interest: the authors report no conflicts of interest.

Contributions: GM collected the human samples and the information from each patient; EA helped write the manuscript; DC tested the human samples; YT designed and supervised the whole process; AP made the final revision check.

Received for publication: 9 August 2011.

Revision received: 11 October 2011.

Accepted for publication: 28 October 2011.

This work is licensed under a Creative Commons Attribution NonCommercial 3.0 License (CC BYNC 3.0).

(C) Copyright G. Minadakis et al., 2011

Licensee PAGEPress, Italy

Infectious Disease Reports 2011; 3:e15

doi:10.4081/idr.2011.e15

immunofluorescence assay (IFA) for IgM and IgG antibodies against $B$. henselae (FOCUS, USA) according to the manufacturer's recommendations. Sera were considered positive in cases of: a) a 4-fold increase in IgM and/or IgG antibodies in two consecutive samples; or b) titers of IgM antibodies over 1/80 and or titers of IgG antibodies over 1/512 in a single sample.

\section{PCR assays}

DNA was extracted using a QIAamp tissue kit (QIAGEN, Hilden, Germany). DNA samples were stored at $-20^{\circ} \mathrm{C}$ until further processing. PCR amplifications were carried out using the primer pair BartoITSF and BartoITSR targeting the intergenic spacer region of Bartonella spp.. ${ }^{9}$ Up to 10 samples were tested along with negative controls (DNA from non-infected lymph nodes and sterile water) and a positive control (DNA from B. elizabethae).

\section{Culture}

Lymph nodes and blood samples were cultured on blood Columbia agar plates, incubated at $37^{\circ} \mathrm{C}$ in an atmosphere of $5 \% \mathrm{CO}_{2}$, and examined weekly for growth during a 2-month period, as previously described. ${ }^{10}$

\section{Statistical analysis}

Data were analyzed using the SPSS v.16 software. ANOVA and Student's t-test were used taking $\mathrm{P} \leq 0.05$ to be significant. 


\section{Results}

Overall, we tested 507 serum samples and we found $56(11 \%)$ cases of CSD (Table 1). In 15 patients a different diagnosis was made other than CSD. Bartonella henselae cross reactions with other microorganisms or even with cancer, lymphoma etc. may occasionally be misleading. By IFA, Bartonella positive results were obtained for 54 (10.6\%) patients. PCR assay was positive for 2 patients; one had a $B$. henselae positive PCR in lymph node and the other a positive PCR in the whole blood sample. For both these 2 patients IFA was negative. No isolates were obtained from the culture of the lymph nodes and blood samples.

Eight (14.2\%) CSD cases were recorded in $2005,13(23.2 \%)$ in 2006, 20 (35.7\%) in 2008 and 15 (26.7\%) in 2008. Positive CSD cases were plotted for each month (Figure 1) to identify seasonal distributions of CSD from 2005 through 2008. Monthly mean numbers of CSD were lowest from February through April, followed by significant increases during May to September $(\mathrm{P}=0.002)$ and during December to January $(P=0.01)$. The age distribution of the 56 confirmed cases ranged from nine months to 73 years. Ages were categorized according to the official age distribution: infants, $0-3$ years; children, 4-15 years; adults, over 15 years (Figure 1). Significantly more CSD cases $(62.5 \%, 35$ of 56$)$ were reported in children than in infants and adults $(\mathrm{P}<0.05)$. No statistically significant difference was calculated in terms of gender (male $42.6 \%$, female 57.1\%).

Epidemiological data revealed that contact with cats was reported in 11 (19.6\%) of the confirmed cases. The clinical features of CSD were local lymphadenopathy (76.8\%) and fever (71.4\%) usually persisting for a few days. In 13 cases (23.2\%) lymphadenopathy without the presence of fever was recorded. Other symptoms such as headache, malaise, arthalgias, myalgias, anorexia and vomiting were reported less often (Table 2). Twenty (35.7\%) patients presented slight anemia ( $\mathrm{Hb}<12$ $\mathrm{g} / \mathrm{dL}$ ) on hospitalization. Leukocytosis was recorded in 15 (37.5\%) patients, with levels ranging from $11 \times 10^{9} / \mathrm{L}$ to $23 \times 10^{9} / \mathrm{L}$. Platelet levels were within normal range in all patients. Complications due to infection by $B$. henselae were observed in 4 patients. Of those, 2 women suffered from sudden vision loss without the co-existence of other clinical symptoms. Vision was restored post treatment. One patient suffered from enlargement of liver and spleen of up to $1.5 \mathrm{~cm}$ together with enlargement of the mesothoracic lymph nodes, all of which receded upon drug therapy. A 1.5 year old infant developed pneumonia together with encephalopathy, hydrocephalus and Guillan Barre syndrome. ${ }^{11}$

Forty patients (71.4\%) received antibiotic therapy; 13 patients (15.7\%) were treated with ciprofloxacin, 12 patients (15\%) with chloramphenicol and one (1.8\%) with doxycycline. Additionally, 9 patients (10.8\%) received a combination of doxycycline and chloramphenicol, while 23 patients $(27.7 \%)$ received doxycycline with ciprofloxacin. All patients receiving antibiotic treatment fully recovered.

\section{Discussion}

In a previous seroepidemiological study conducted in 491 samples of healthy blood donors in Crete, a $21.6 \%$ seroprevalence against $B$. henselae was recorded. ${ }^{12}$ In the current study, we identified 56 cases of CSD during a 3 -year period. Our results were based on clinical and epidemiological data and on molecular and serological assays. Our PCR assay had been previously validated for the diagnosis of CSD in lymph nodes biopsies. ${ }^{1,9}$ Although culture of lymph nodes and blood samples was systematically performed, we did not obtain Bartonella isolates. Indeed, $B$. henselae is not frequently grown from human lymph nodes and only in a few cases has $B$. henselae been isolated from patients with CSD. ${ }^{1,13}$

Most of our cases were children. This agrees with other studies that have shown that most cases of CSD occur in children and young adults. ${ }^{14}$ However, a small number of patients with CSD aged over 60 years were also identified and characterized. In a previous study in Israel, Ben Ami et al. found that CSD affects elderly subjects as well as other age groups. ${ }^{15}$ In this study, the most cases were recorded during the summer period with another peak recorded in winter. In the USA, it was found that CSD

Table 1. Distribution of IgG titers in each age group.

\begin{tabular}{lrccc} 
Iiter of $\lg G$ antibodies & $0-20$ & $21-30$ & $31-40$ & $\geq 51$ \\
0 & 14 & 0 & 1 & 0 \\
$1 / 64$ & 1 & 0 & 0 & 0 \\
\hline $1 / 128$ & 5 & 0 & 0 & 0 \\
$1 / 256$ & 6 & 0 & 0 & 0 \\
$1 / 512$ & 13 & 4 & 2 & 1 \\
$1 / 1024$ & 6 & 0 & 1 & 1 \\
\hline $1 / 2048$ & 1 & 0 & 0 & 0 \\
\hline
\end{tabular}

Table 2. Signs and symptoms recorded among the 56 confirmed Cat-scratch disease cases.

\begin{tabular}{lcc} 
Signs - symptoms & No & $\%$ \\
Fever & 40 & 71,4 \\
Lymphadenopathy & 43 & 76,8 \\
Neck & 20 & 35,7 \\
Inguinal & 11 & 19,6 \\
Armpit & 12 & 21,4 \\
\hline Hepato-splenomegaly & 7 & 12,5 \\
Arthalgia & 5 & 8,9 \\
\hline Malaise & 5 & 8,9 \\
Rash & 5 & 8,9 \\
Maculopapular & 1 & 1,8 \\
Papular & 1 & 1,8 \\
Erythema nodosum & 2 & 3,6 \\
\hline Headache & 4 & 7,1 \\
Impaired vision (vision loss, squint) & 8 & 14,3 \\
\hline Myalgia & 2 & 3,6 \\
Anorexia & 3 & 5,3 \\
\hline Vomiting & 2 & 3,6 \\
Pericarditis & 2 & 3,8 \\
\hline Arrhythmias & 2 & 8,9 \\
Confusion, loss of conscience & 5 & 10,7 \\
\hline Hematuria & 6 & 3,5 \\
Stiff neck & 2 & 3,6 \\
\hline
\end{tabular}


presented a seasonal variation from September through January ${ }^{16}$ and most CSD-associated hospitalizations for patients under the age of 18 years occurred between July and October. ${ }^{17}$ In addition, in Japan, CSD mainly occurred in the fall and winter months, as most cases occurred between September and December. ${ }^{18}$ In France, a reduced incidence was recorded during spring with an increase in autumn and peaks in December. ${ }^{19}$ The difference observed in the United States may be attributed to the fact that it is a large country with diverse climates. As far as Crete is concerned, the fact that cats have three reproduction periods rather than two may well explain the presence of the peak recorded during winter.

People in Crete do not keep pets like cats indoors and probably the increase of CSD cases during the summer is explained by the increase in outdoor activity and greater contact with pound or street cats. Moreover, the higher seroprevalence of $B$. henselae in the pet cat population in warm, humid climates than in cats in cold, dry climates is explained because $C t$. felis is more common in warmer climates. ${ }^{20}$ This can be explained because the environmental conditions of temperature and relative humidity are the two most important factors for the successful reproduction, development and survival of fleas..$^{21}$ As a result, cats present more fleas during the summer. ${ }^{22}$

Local lymphadenopathy and fever were the most common symptoms in our study. Uncommon clinical signs were malaise, fatigue, myalgia and arthralgia, and hepatosplenomegaly. In patients with classic CSD, lymphadenopathy is the most common symptom and occurs most frequently in the axillary and epitrochlear nodes (46\%), head and neck (26\%), and the groin (17.5\%). ${ }^{23}$ Most patients with typical CSD remain afebrile and in a series of 1,200 cases the disease was considered mild in over 75\%; $41 \%$ were afebrile, $31 \%$ had fever below $39^{\circ} \mathrm{C}, 19 \%$ had fever by touch and only $9 \%$ had fever over $39^{\circ} \mathrm{C} . .^{24}$ In another series of 1,722 patients with CSD, almost one-half had lymphadenopathy only. ${ }^{25}$ Systemic or severe disease can complicate

\section{A No of confirmed cases per month}

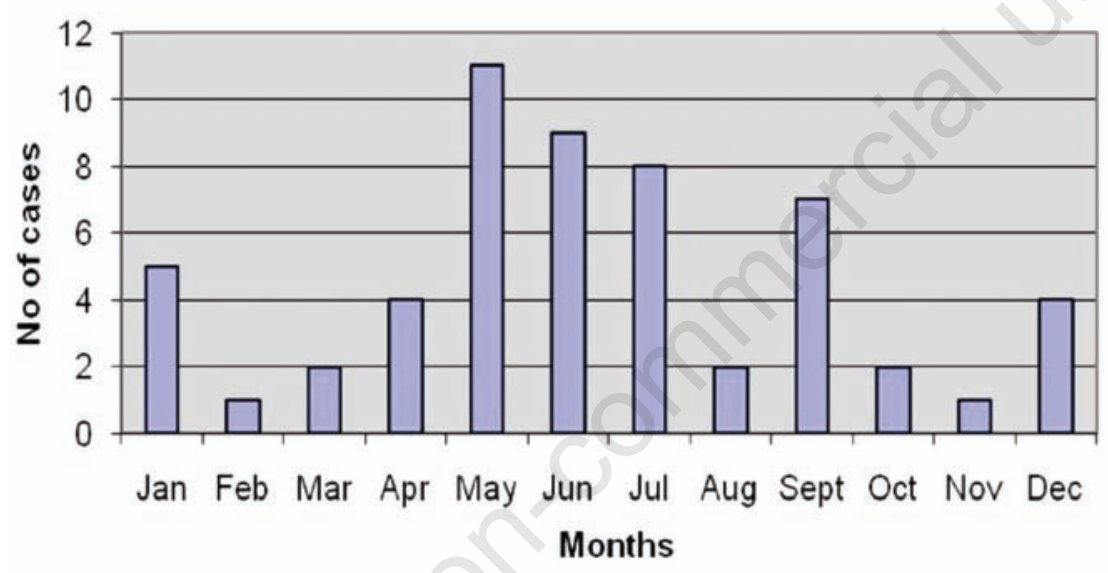

B

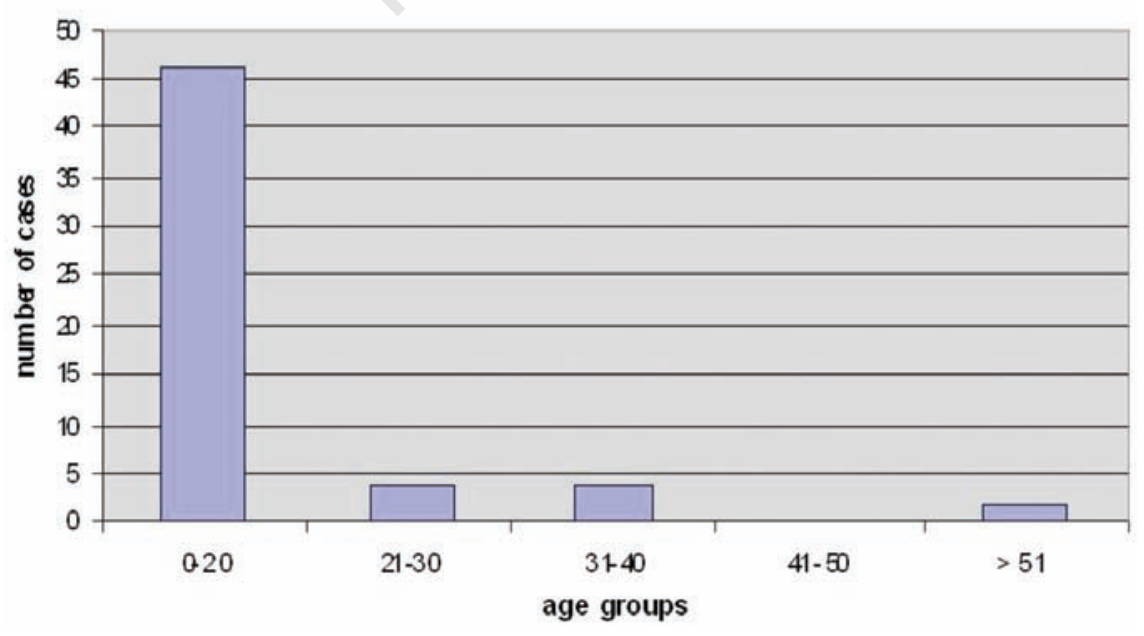

Figure 1. Distribution of Cat-scratch disease cases by (A) month and (B) age.
CSD in $5-14 \%$ of cases. ${ }^{23}$ Atypical presentations include prolonged fever (over 2 weeks), malaise, fatigue, myalgia and arthralgia, weight loss, splenomegaly, and Parinaud's oculoglandular syndrome. ${ }^{26}$ Encephalopathy and neuroretinitis are less common complications of CSD. ${ }^{26}$

In conclusion, we proved that CSD is prevalent in Crete and is seasonal starting in May and finishing in September. The increase in outdoor activity and in $\mathrm{Ct}$. felis during the summer months probably explain this seasonality. The differential diagnosis of CSD is often misleading and more serious conditions (mycobacterial or fungal infections, lymphoma, melanoblastoma, carcinoma) are often considered. These may entail unnecessary, expensive, and often invasive evaluations and protracted hospital stays. Because of this, accurate diagnosis is of critical importance. Physicians need to inquire about recent contact with an animal, especially felines, or injury from an animal, when a patient presents with or without lymphadenopathy.

\section{References}

1. Rolain JM, Lepidi H, Zanaret M, et al. Lymph node biopsy specimens and diagnosis of cat-scratch disease. Emerg Infect Dis 2006;12:1338-44.

2. Angelakis E, Pulcini C, Waton J, et al. Scalp eschar and neck lymphadenopathy caused by Bartonella henselae after Tick Bite. Clin Infect Dis 2010;50:549-51.

3. Angelakis E, Lepidi $\mathrm{H}$, Canel $\mathrm{A}$, et al. Human case of Bartonella alsatica lymphadenitis. Emerg Infect Dis 2008;14: 1951-3.

4. Chomel BB, Kasten RW, Floyd-Hawkins K, et al. Experimental transmission of Bartonella henselae by the cat flea. J Clin Microbiol 1996;34:1952-6.

5. Carithers HA. Cat scratch disease associated with an osteolytic lesion. American Journal of Diseases of Children 1983; 137:968-70.

6. Ridder GJ, Boedeker CC, Technau-Ihling K, et al. Role of cat-scratch disease in lymphadenopathy in the head and neck. Clinical Infectious Diseases 2002;35:643-9.

7. Angelakis E, Edouard S, La SB, Raoult D. Bartonella henselae in skin biopsy specimens of patients with cat-scratch disease. Emerg Infect Dis 2010;16:1963-5.

8. Zangwill KM, Hamilton DH, Perkins BA, et al. Cat scratch disease in Connecticut Epidemiology, risk factors, and evaluation of a new diagnostic test. New England Journal of Medicine 1993;329:8-13.

9. Angelakis E, Roux V, Raoult D, Rolain JM. Real-time PCR strategy and detection of 
bacterial agents of lymphadenitis. Eur J Clin Microbiol Infect Dis 2009;28:1363-8.

10. La Scola B, Raoult D. Culture of Bartonella quintana and Bartonella henselae from human samples: a 5-year experience (1993 to 1998). J Clin Microbiol 1999;37: 1899-905.

11. Mantadakis E, Spanaki AM, Psaroulaki A, et al. Encephalopathy complicated by Guillain-Barre syndrome and hydrocephalus and associated with acute Bartonella quintana infection. Pediatr Infect Dis J 2007;26:860-2.

12. Minadakis G, Chochlakis D, Kokkini S, et al. Seroprevalence of Bartonella henselae antibodies in blood donors in Crete. Scand J Infect Dis 2008;1-2.

13. Fournier PE, Robson J, Zeaiter Z, et al. Improved Culture from Lymph Nodes of Patients with Cat Scratch Disease and Genotypic Characterization of Bartonella henselae Isolates in Australia. J Clin Microbiol 2002;40:3620-4.

14. Hamilton DH, Zangwill KM, Hadler JL, Cartter ML. Cat scratch disease -
Connecticut, 1992-1993. J Infect Dis 1995; 172:570-3.

15. Ben Ami R, Ephros M, Avidor B, et al. Catscratch disease in elderly patients. Clin Infect Dis 2005;41:969-74.

16. Jackson LA, Perkins BA, Wenger JD. Cat scratch disease in the United States : an analysis of three national databases. Am J Public Health 1993;83:1707-11.

17. Reynolds MG, Holman RC, Curns AT, et al. Epidemiology of cat-scratch disease hospitalizations among children in the United States. Pediatr Infect Dis J 2005;24:700-4.

18. Tsukahara M. Cat scratch disease in Japan. J Infect Chemother 2002;8:321-5.

19. Sanguinetti-Morelli D, Angelakis E, Richet H, Davoust B, Rolain JM, Raoult D. Seasonality of cat-scratch disease, France, 1999-2009. Emerg Infect Dis 2011;17:705-7.

20. Chomel BB, Abbott RC, Kasten RW, et al. Bartonella henselae prevalence in domestic cats in California: Risk factors and association between bacteremia and antibody titers. J Clin Microbiol 1995;33:2445-50.

21. Dryden MW, Rust MK. The cat flea: biology, ecology and control. Vet Parasitol 1994;52:1-19.

22. Farkas R, Gyurkovszky M, Solymosi N, Beugnet $F$. Prevalence of flea infestation in dogs and cats in Hungary combined with a survey of owner awareness. Med Vet Entomol 2009;23:187-94.

23. Carithers HA. Cat-scratch disease : an overview based on a study of 1200 patients. Am J Dis Child 1985;139:1124-33.

24. Carithers HA, Carithers CM, Edwards RO. Cat scratch disease: its natural history. J Amer Med Assoc 1969;207:312-6.

25. Margileth AM. Antibiotic therapy for cat scratch disease: clinical study of therapeutic outcome in 268 patients and a review of the literature. Pediatr Infect Dis J 1992; 11:474-8.

26. Bass JW, Vincent JF, Person DA. The expanding spectrum of Bartonella infections: II. Cat scratch disease. Pediatric Infectious Disease Journal 1997;16(2): 163-79. 